\title{
Association of Infantile Hemangiomas and Retinopathy of Prematurity: Analysis of the Multicenter KID
}

\author{
Nilesh Dankhara Renjithkumar Kalikkot Thekkeveedu Jaimin Patel \\ Jagdish Desai \\ Department of Pediatrics, University of Mississippi Medical Center, Jackson, MS, USA
}

\section{Keywords}

Infantile hemangioma - Retinopathy of prematurity ·

Gender Race Premature infant

\begin{abstract}
Introduction: Retinopathy of prematurity (ROP) and infantile hemangiomas $(\mathrm{IHs})$ both have similar proposed pathophysiological mechanisms. IH is more common in preterm than term infants. Hypoxia-induced mediators like vascular endothelial growth factor have been found elevated in children with hemangiomas. The aim of our study was to determine if there is an association between ROP and IH in preterm infants and to investigate racial/ethnic and gender differences of ROP and IHs in this cohort. Methods: We accessed the national multicenter Kids' Inpatient Database (KID) Healthcare Cost and Utilization Project (HCUP) including admissions at age $\leq 28$ days. Eligible infants were identified by using ICD-9 codes of ROP and IH in infants with gestational age $(G A) \leq 32$ weeks and/or birth weight $\leq 1,500$ g during the years 2003, 2006, 2009, and 2012. A weight-based analysis was performed using SAS Enterprise Guide 7.1 for complex sample design. Results: In the cohort of 1,068,502 eligible infants, the prevalence of $\mathrm{IH}$ was 4.7 per 1,000 preterm ad-
\end{abstract}

missions ( $<32$ weeks). ROP prevalence was $16 \%$ for $\mathrm{GA} \leq 26$ weeks, $12.5 \%$ for GA $27-30$ weeks, and $2.7 \%$ for GA 31-32 weeks. IH was significantly higher in infants with ROP; this relationship was consistent among all stages of ROP. Regression analysis showed that females are at increased risk of $\mathrm{IH}$ with ROP compared to males (adjusted odds ratio [aOR]: 2.00 [1.85-2.56]). White non-Hispanic premature infants had an increased risk of $\mathrm{IH}$ with concomitant ROP compared to both African American (aOR: 3.9 [2.63-4.76]) and Hispanic (aOR: 1.2 [1.14-1.38]) infants. However, African American infants had an increased risk of ROP compared to white non-Hispanic infants (aOR: 1.16 [1.07-1.14]). These genders and racial/ ethnic disparities were consistent among GA categories. Conclusions: To our knowledge, this is the largest cohort based on a national multicenter database comparing an association between ROP and IH. A strong association between $\mathrm{ROP}$ and IH may suggest similar risk factors and/or pathophysiology. A further role of genetic factors could explain racial/ethnic differences in both conditions despite similar pathogenesis. These findings may open up new bases of research for management and prevention strategies.

(c) 2022 The Author(s).

Published by S. Karger AG, Basel karger@karger.com www.karger.com/bmh

Karger ${ }^{\prime \prime}$

BOPEN ACCESS
C) 2022 The Author(s)

Published by S. Karger AG, Basel

This is an Open Access article licensed under the Creative Commons Attribution-NonCommercial-4.0 International License (CC BY-NC) (http://www.karger.com/Services/OpenAccessLicense), applicable to the online version of the article only. Usage and distribution for commercial purposes requires written permission.
Correspondence to:

Jagdish Desai, jdesai@umc.edu 


\section{Introduction}

Retinopathy of prematurity ( $\mathrm{ROP}$ ) is defined as a vasoproliferative disorder affecting the retina of preterm infants, and it is the leading cause of blindness in children $[1,2]$. During normal development, retinal blood vessels develop from the optic disc center toward the edge of the retina, known as ora serrata. ROP develops in the immature retina, where the retinal blood vessels have not reached the ora serrata $[3,4]$. Prematurity and low birth weight are important risk factors for ROP development, with a significantly higher incidence in infants born at $<32$ weeks of gestational age (GA) or birth weight $<1,251$ g. Incidence and severity of ROP has an inverse relationship with prematurity and birth weight [5]. Extreme low birth weight infants are at much higher risk of ROP than very low birth weight infants [1]. Based on the revised international classification of ROP in 2005, it is classified into 5 stages (stage 1-5) based on the abnormal vascular response at the junction of the vascularized and avascular retina [3]. Our understanding of the pathophysiology of ROP has improved significantly in the last 3 decades, and many studies have shown that insulin-like growth factor 1 (IGF-1) and vascular endothelial growth factor (VEGF) play an important role in the pathogenesis of ROP. VEGF, an important cytokine for neovascularization, expression is enhanced by hyperoxia in the retina. IGF-1 has also been shown to be an important factor for VEGF signals to induce angiogenesis $[6,7]$.

Infantile hemangiomas (IHs) are benign vascular tumors characterized by excessive proliferation of immature microvessels seen in infants and young children [8]. They are considered as the most common vascular tumor in infants $[9,10]$, seen in about $2-10 \%$ of infants. They can vary in size from small to large, life-threatening lesions $[11,12]$. It is reported more commonly in female and non-Hispanic white infants [12-15]. An increased incidence is also associated with prematurity [15], low birth weight, multiple gestation, advanced maternal age, pre-eclampsia $[12,15]$, and placental abnormalities $[15$, 16]. About $15-60 \%$ of these lesions are present at birth, becoming apparent in the first few weeks of life $[9,11,17]$. Almost half of these are on the head and neck, although they can arise anywhere on the body, like internal organs such as the liver, along the mucosal lining of the gastrointestinal tract, and within the central nervous system [11]. They have a characteristic proliferative phase (endothelial cell proliferation), which lasts on an average of 6-18 months, followed by a plateau period (months to years) with gradual involution until 3-9 years $[10,18,19]$.
An association between ROP and $\mathrm{IH}$ has always intrigued clinicians. Such an association was first reported in the early 1950s by Reese et al. [20]. Later in that decade, a couple of other researchers also have reported a similar association between the 2 entities [21,22]. More recently, some other studies explored the existence of such association between the 2 pathologies $[8,19]$. Most of these studies are either old, not representing the neonatal population that we currently encounter, or smaller sample sizes making it difficult to extrapolate these results into larger populations. Hence, there is a need to validate this association from a large representative population-based sample in the USA.

The primary aim of this study was to explore the association between ROP and IH based on the theory that they possibly have common underlying pathogenesis. We also investigated the demographic characteristics like racial/ethnic and gender differences of ROP and IHs in this cohort.

\section{Method}

We analyzed data collected as part of the administrative Kids' Inpatient Database (KID). The Healthcare Cost and Utilization Project, administered by the Agency for Healthcare Research and Quality, produces the National Inpatient Sample and its pediatricsonly version, KID [23]. This all-payer database annually collects information from hospital administrative discharge (i.e., billing) records from about 4,000 hospitals across 44 states in the USA of various levels (primary to tertiary), settings (university academic and general), and types of insurance (public and private). About 100 data elements (International Classification of Disease, Ninth Revision, Clinical Modification [ICD-9-CM] diagnoses, Current Procedural Terminology procedures, demographic and hospital characteristics, discharge status, LOS, severity, and comorbidity measures) for each hospital stay are included from the discharge abstract. KID data are available every 3 years between 1997 and 2016 and have a random sample of $10 \%$ of uncomplicated in-hospital births and $80 \%$ of other pediatric discharge codes from participating institutions. The KID allows calculation of national estimates with sampling error by using discharge weight, cluster (hospital), and stratum (region) variables in the analyses, in which the basic unit is a patient discharge rather than an individual patient.

Case abstraction was done using ICD-9 discharge codes for ROP (362.20-362.29), IH (228.00, 228.01, 228.09), prematurity defined by birth GA $\leq 32$ weeks (765.21-765.26, V21.31-V21.33), and low birth weight defined by birth weight $<1,500 \mathrm{~g}$ (765.01$765.05,765.11-765.16)$. As ROP exams are not routinely done for GA > 32 weeks, including GA > 32 weeks may confound our result, and so they were excluded. Infants admitted within $\leq 28$ days of birth, inborn or transferred in, during the years 2003, 2006, 2009, and 2012 were included in the cohort. Clinical data were abstracted for the period between admission and discharge, transfer, or death. Stillbirths (ICD-9-CM V32, V35, or V36) and congenital anomalies were excluded. Infants with a final disposition coded as transfer were excluded to minimize duplication. All ICD and DRG 


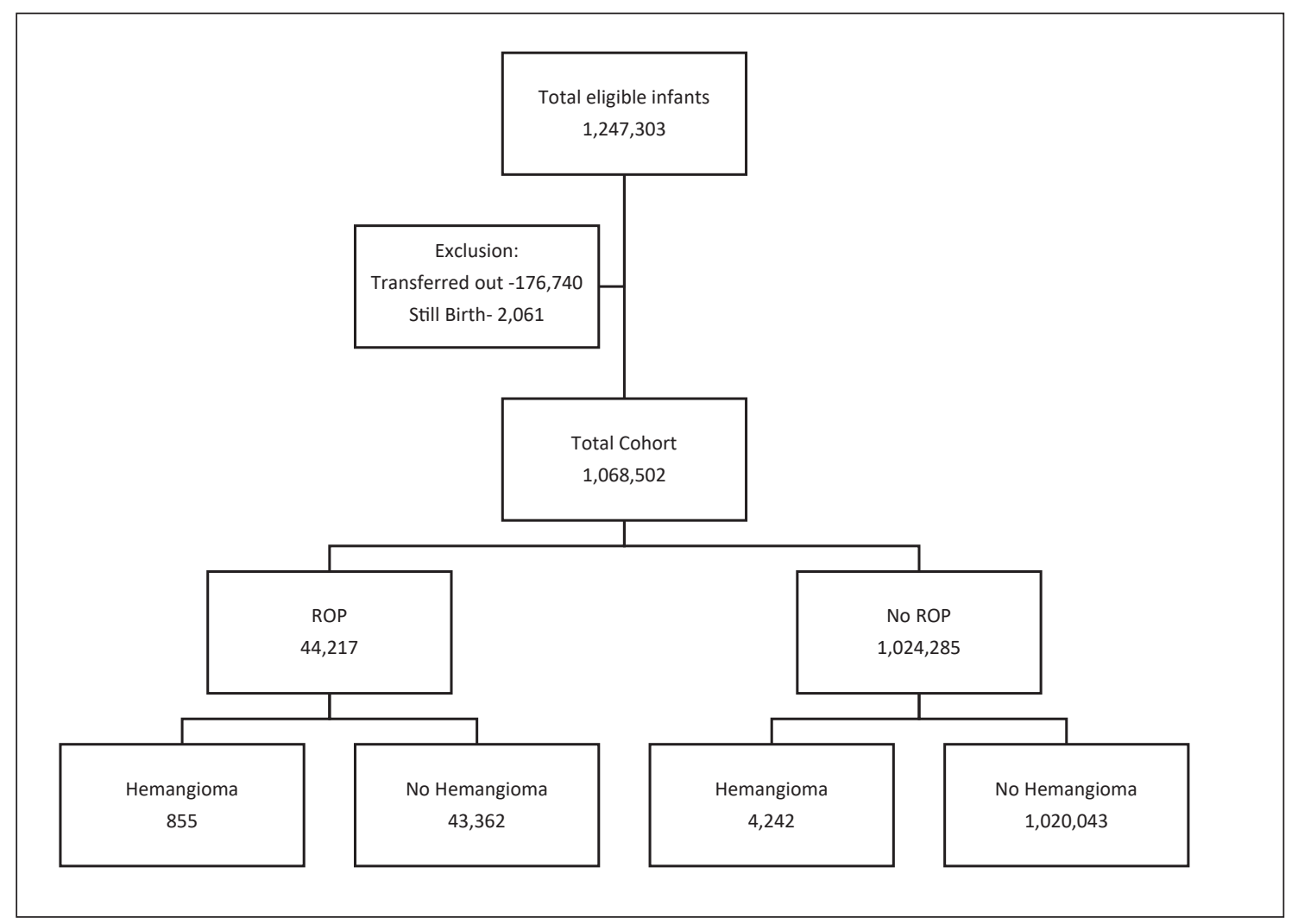

Fig. 1. Cohort extraction.

codes used for cases extraction are in online supplementary Table 1 (see www.karger.com/doi/10.1159/000521413 for all online suppl. material).

\section{Statistical Analysis}

Baseline characteristics were compared by using $\chi^{2}$ linear-bylinear association for categorical variables and 1-way ANOVA for continuous variables; alpha was set at 0.01 (Bonferroni correction) for statistical significance. Demographic, clinical, and hospital characteristics and prematurity-associated comorbidity indicators (bronchopulmonary dysplasia [BPD]) were assessed as independent associations with ROP using adjusted logistic regression models. Variables with a $p$ value of 0.1 on univariate analysis were selected as candidates for the final model and were assessed for collinearity and interaction. ORs with 95\% CIs were calculated for each association. Data were analyzed with SAS software, version 9.4 (SAS Institute, Cary, NC, USA), and all tests were 2-tailed.

\section{Results}

A total of 1,068,502 eligible neonates were included in the data analysis as shown in Figure 1. In this cohort, the prevalence of IH was 4.7 per 1,000 preterm ( $<32$ weeks GA) admissions. Baseline characteristic of our study population includes demographics, GA, hospital characteristics, and BPD status as shown in Table 1. If GA was specified, it was highest among 27-30 weeks of GA category (31\%). IH was more common in females, white race, in southern geographical region, and in urban teaching hospitals. It was also more common in presence of congenital anomalies and having diagnosis of BPD during the hospital stay.

The overall prevalence of ROP in all GA ( $<32$ weeks) was 41.3 per 1,000 preterm ( $<32$ weeks GA) admissions, and it was $16 \%$ for GA $\leq 26$ weeks, $12.5 \%$ for GA $27-30$ weeks, and $2.7 \%$ for GA $31-32$ weeks. IHs were significantly higher (aOR = 3.13, CI: 2.22-3.40) in infants with ROP after adjusting for GA, sex, race, location of the hospital, and teaching status of the hospital; this relationship was consistent among all stages of ROP as shown in Table 2 . The presence of IH among ROP infants who required surgical intervention versus who did not require surgical intervention was not statistically significant $(1.8 \%[67 / 3,623]$ vs. $1.9 \%[787 / 39,739], p=0.28)$. Regression analysis showed that females are at increased risk of IH with ROP compared to males (aOR: 2.00, CI: 1.852.56 ) as shown in Figure 2. Non-Hispanic white prema- 
Table 1. Baseline characteristics

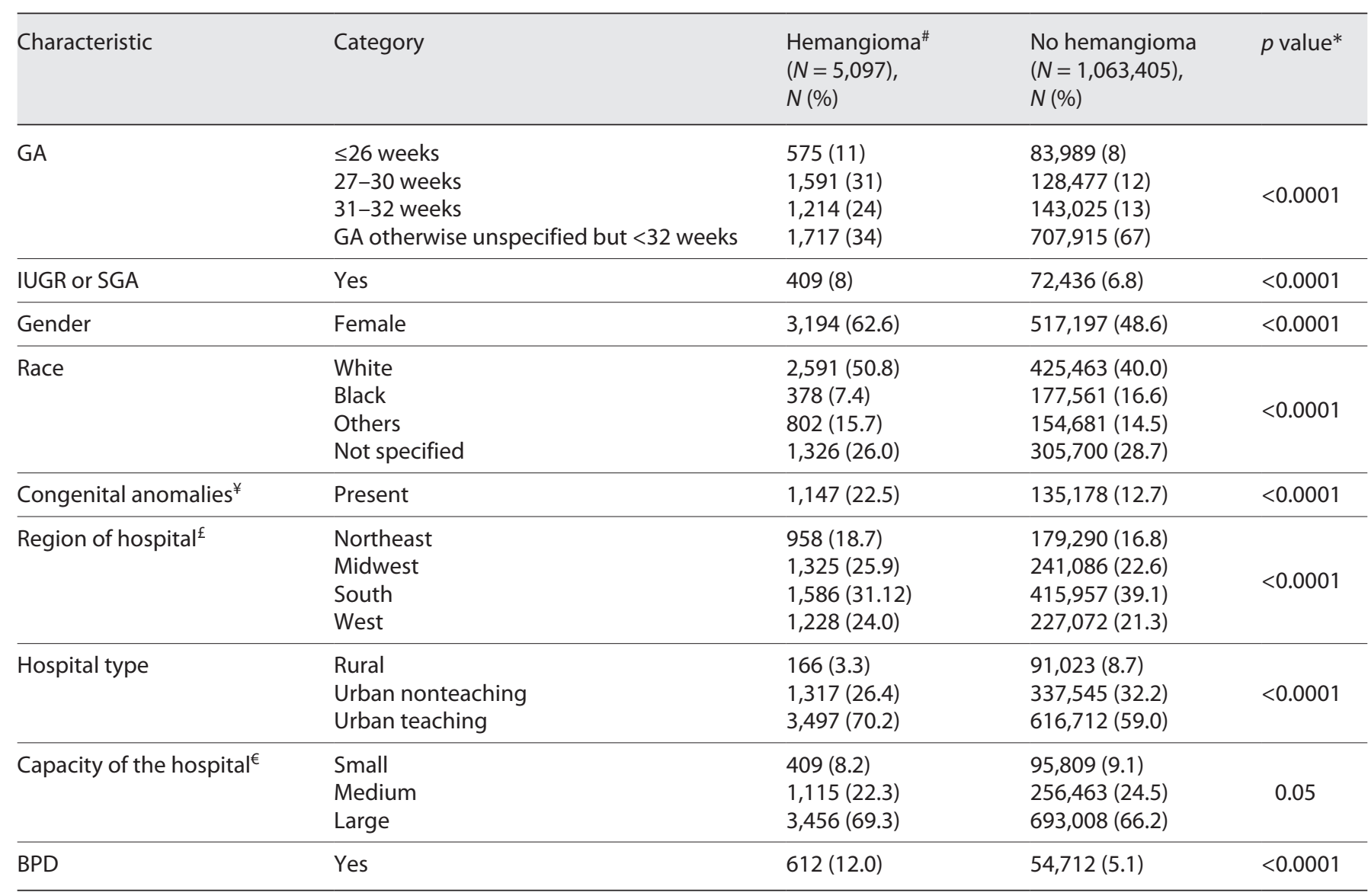

IUGR, intrauterine growth restriction; SGA, small for gestational age; BPD, bronchopulmonary dysplasia. * $p$ value significant at a $=0.05$ level by the $x^{2}$ test. \#Prevalence of $\mathrm{IH}: 5,097$ (total IH cases)/1,068,502 (total population at risk) $\times 1,000=4.7$ cases per 1,000 preterm ( $<32$ weeks GA) live birth admissions. ${ }^{¥}$ ICD codes for congenital anomalies are described in online supplementary Table $1 .{ }^{\ddagger}$ Hospital regions: this is an important stratifier because practice patterns have been shown to vary substantially by regions. ${ }^{€}$ Capacity categories are based on the number of hospital beds and are specific to the hospital's location and teaching status. Capacity of the hospital's categories is defined using region of the USA and the urban-rural designation of the hospital, in addition to the teaching status (https://www.hcup-us.ahrq.gov/ $\mathrm{db} /$ vars/hosp_bedsize/nisnote.jsp).

ture infants had an increased risk of $\mathrm{IH}$ with concomitant ROP compared to both African American (aOR: 3.9, CI: 2.63-4.76) and Hispanic (aOR: 1.2, CI: 1.14-1.38) infants. However, African American infants had an increased risk of ROP compared to non-Hispanic white infants (aOR: 1.16, CI: 1.07-1.14). These genders and racial/ethnic disparities were consistent among GA categories as well.

\section{Discussion}

We have analyzed the Kids' Inpatient Database (KID) data, a large population-based administrative database compiled by the HCUP of AHRQ. We analyzed the data during the years 2003, 2006, 2009, and 2012. A total of $1,068,502$ eligible neonates were analyzed in our study, and we adjusted our data for GA, sex, race, location of the hospital, and teaching status of the hospital. This report is one of the largest of its kind, representing $>4,000$ hospitals and $>22$ million discharge records over the last 2 decades.

We found a significant association between $\mathrm{IH}$ and ROP in our study. This is similar to Praveen et al. [19], who studied about 400 babies from a single center in the USA and reported a significant association between $\mathrm{IH}$ and ROP after adjusting for GA and postnatal steroid use. They also found a higher incidence of $\mathrm{IH}$ with higher stages of ROP [19]. We did not find any such change in 
Table 2. Hemangioma and ROP

\begin{tabular}{|c|c|c|c|c|c|}
\hline \multirow[t]{2}{*}{ ROP\# } & \multicolumn{2}{|c|}{ Hemangioma, $N(\%)$} & \multirow[t]{2}{*}{$p$ value* } & \multirow{2}{*}{$\begin{array}{l}\text { Unadjusted OR } \\
(95 \% \mathrm{Cl})\end{array}$} & \multirow{2}{*}{$\begin{array}{l}\text { Adjusted OR }{ }^{£} \\
(95 \% \mathrm{Cl})\end{array}$} \\
\hline & yes & no & & & \\
\hline \multicolumn{6}{|c|}{ Total ROP } \\
\hline Yes & $825(2.5)$ & $32,555(97.5)$ & $<0.0001$ & $6.12(5.55-6.75)$ & $3.13(2.22-3.40)$ \\
\hline No & $4,272(0.4)$ & $1,030,850(99.6)$ & & & \\
\hline \multicolumn{6}{|c|}{ ROP stage $1-2$} \\
\hline Yes & $606(2.5)$ & $23,231(97.5)$ & $<0.0001$ & $6.04(5.40-6.70)$ & $3.00(2.62-3.33)$ \\
\hline No & $4,491(0.4)$ & $1,040,174(99.5)$ & & & \\
\hline \multicolumn{6}{|c|}{ ROP stage $3-5$} \\
\hline Yes & $78(2.3)$ & $3,302(97.7)$ & $<0.0001$ & $4.50(3.80-6.50)$ & $2.70(2.04-3.52)$ \\
\hline No & $5,020(0.5)$ & $1,060,103(99.5)$ & & & \\
\hline \multicolumn{6}{|c|}{ ROP stage NOS } \\
\hline Yes & $148(2.3)$ & $6,200(97.7)$ & $<0.0001$ & $5.10(4.20-6.20)$ & $2.50(2.03-3.01)$ \\
\hline No & $4,949(0.5)$ & $1,057,205$ (99.5) & & & \\
\hline
\end{tabular}

Missing values in the regression model are eliminated. As regression analysis for ROP stages (ROP stage 1-2, ROP stage 3-5, and ROP stage NOS) are run separately for each category, numbers will not add up to the total ROP category due to missing values. For Figure 1, numbers represent the total cohort, and in Table 2, numbers represent the $N$ entered in the regression analysis. If any variable entered in the model has missing value, that case will be eliminated from regression analysis. That is the reason, numbers look different in Figure 1 and Table 2. NOS, not otherwise specified. ${ }^{*} p$ value significant at $a=0.05$ level by the $x^{2}$ test. ${ }^{\ddagger}$ Binary logistic regression analysis: adjusted for GA, sex, race, BPD, geographic location of hospital (northeast, midwest, south, and west), size of the hospital (small, medium, and large), and teaching status of the hospital (rural, urban nonteaching, and urban teaching). \# Prevalence of ROP: 44,217 (total ROP cases)/1,068,502 (total population at risk) $\times 1,000=41.3$ cases per 1,000 preterm ( $<32$ weeks $\mathrm{GA})$ live birth admissions.

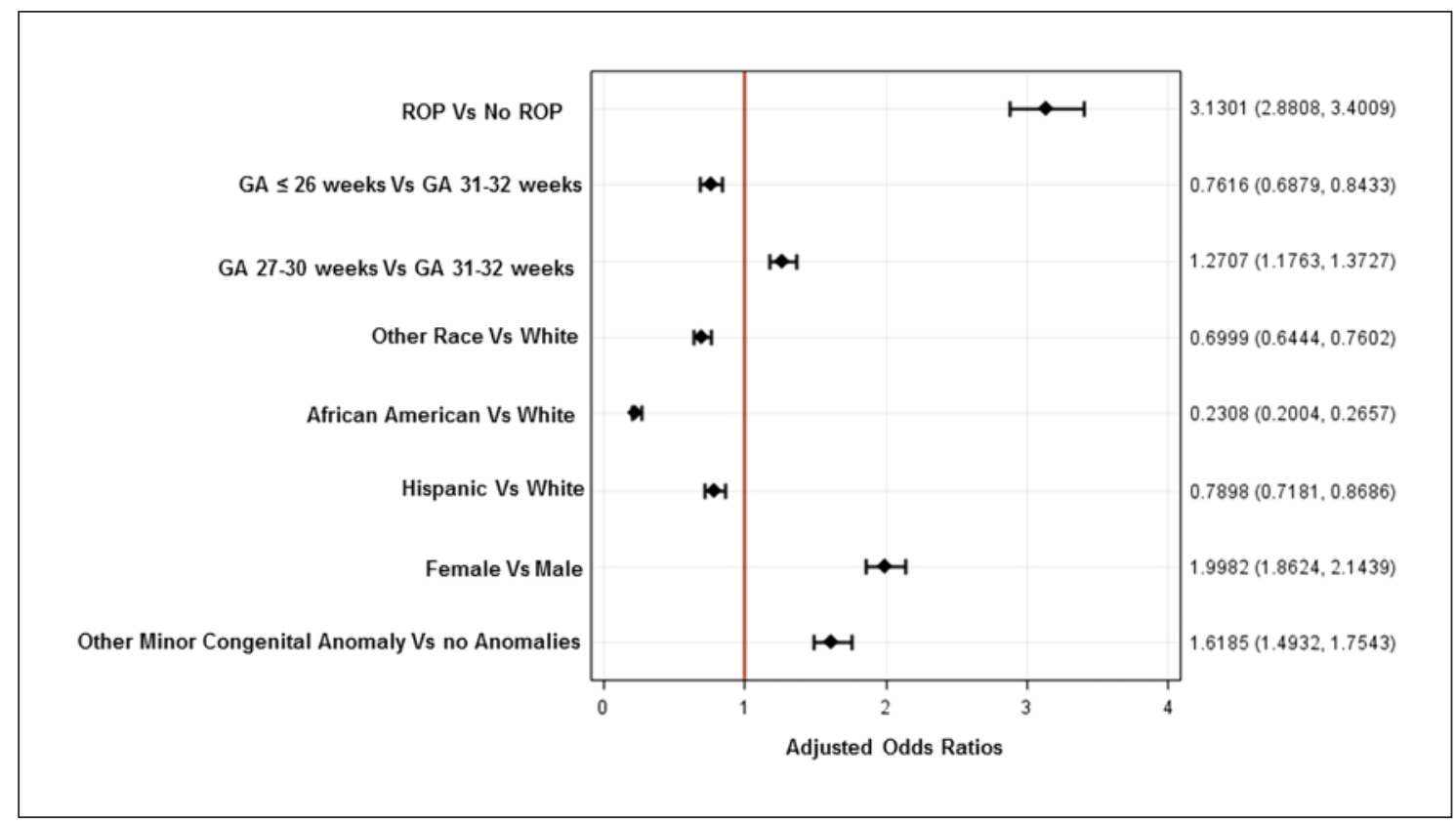

Fig. 2. Forrest plot of IH by regression analysis. aOR (95\% CI) of IH in infants with ROP controlled for GA, race, gender, and other congenital anomalies. 
the incidence of IH with the increasing severity of ROP. Hyland et al. [8] examined the data from about 900 babies born at $<32$ weeks at 2 centers in the USA and Hungary in a retrospective design. Although they reported that they could not find a significant relation to ROP and IH for their 2 centers, in their initial analysis, they found a significant relationship between $\mathrm{IH}$ and ROP through random-effects meta-analysis [8].

The association between ROP and IH may be due to the common underlying mechanism for microvascular proliferation in their development. Although exact pathogenesis remains unknown, local tissue hypoxia, embolization of placental endothelial cells, and increased angiogenic and vasculogenic activity are hypothesized to be the mechanism involved in the pathogenesis of IH [10]. Two major proangiogenic factors are involved during the growth phase: basic fibroblast growth factor and VEGF. The betaadrenergic system may also play a role in their pathogenesis [12, 24, 25]. Hypoxia, increased levels of hypoxia inducible factor-1 (HIF-1), IGF-1, and VEGF, and activation of the beta-adrenergic system are implicated in the pathogenesis of the proliferative phase of ROP as well [24]. ROP and IHs share certain striking features histologically as well, such as active mitotic endothelial fronds or sprouts in the early phase [26]. Beta 2 blockade can inhibit the production of VEGF and is the basis of considering $\beta$-blockers in the treatment of IH and ROP $[27,28]$.

The prevalence of IH in our study was 4.7 per 1,000 preterm admissions. In a study by Amir et al. [29], IH was noted in $12.7 \%$ of preterm infants during the first year of life. Their study was from a relatively small sample size (973 infants) [29]. The increased prevalence in their study may be because the authors looked at the prevalence over the first year of life and could detect some of the lesions that were not present during the birth admission.

Our study found an increased prevalence of $\mathrm{IH}$ and ROP with decreasing GA. Haggstrom et al. [15] have reported an increased prevalence in preterm $(<37$ weeks) and very preterm $(<32$ weeks). They were also more likely to be of low birth weight or very low birth weight [15]. We only included babies with GA $<32$ weeks and found that the incidence of ROP and IH is higher in the cohort with GA <26 weeks compared to GA 27-30 weeks, which was more than GA 31-32 weeks.

In our study, we found that IHs and ROP were twice more common in girls than boys. This is similar to what was reported by Amrock et al. [13], who noted that girls were 1.43 times more likely than boys to get a diagnosis of IH from their analysis of the National Hospital Discharge Survey (NHDS) newborn subset with about
785,042 newborn discharge records. Amir et al. [29] also have noted male:female ratio was 1:1.4 in their study. Haggstrom et al. [15] also have reported a similarly increased prevalence of IH in females at a female-to-male ratio of 2.4:1. The reason for female predominance is unclear. No genetic mutations on the X chromosome have been reported so far. There are reports that IH cells express estrogen receptors. There is a synergistic relationship between estrogen receptor stimulation and hypoxia in epithelial progenitor cell mobilization, a key step in postnatal vasculogenesis [30]. Estrogen has been associated with the induction of endothelial proliferation as well [31]. This may explain the higher incidence of IH in female infants.

We also found an increased odds of both $\mathrm{IH}$ and ROP in non-Hispanic whites compared to African American population and Hispanic population. Similarly, Amrock et al. [13] also have reported that white infants are 1.5, 1.8, and 3.6 times more likely to be diagnosed with $\mathrm{IH}$ than blacks, Asians, and American Indians, respectively. Between 1979 and 2006, they also observed a doubling of IH diagnoses in white newborns [13].

The limitation of this study includes the retrospective design and limitations related to the administrative database. The findings heavily rely on the accurate reporting of the ICD codes and other variables.

\section{Conclusion}

Our results show that ROP and $\mathrm{IH}$ are likely to cooccur in preterm infants, but we need prospective studies to confirm and more clearly define this association. We also need studies to understand both disorders' pathogenesis in detail better to improve our understanding of this association. Further research studies on the biology of vasculogenesis in ROP and IH may help to understand any overlapping mechanisms between ROP and IH.

\section{Statement of Ethics}

Our study was determined to be exempt by the University of Mississippi Medical Center Institutional Review Board. Written informed consent was not required as this was conducted using data from a deidentified national database.

\section{Conflict of Interest Statement}

The authors have no conflicts of interest to declare. 


\section{Funding Sources}

The authors received no funding for the analysis or preparation of this manuscript.

\section{Author Contributions}

J.D. did the statistical analysis with help from J.P. N.D. and R.K.T. wrote the first draft of the manuscript. The manuscript was revised based on feedback and help from J.D. and J.P.

\section{Data Availability Statement}

Data used in this project are available from the HCUP website for purchase https://www.hcup-us.ahrq.gov/. Code segments can be made available upon request to the author.

\section{References}

1 Silverman WA. Missing and unaccounted for. Paediatr Perinat Epidemiol. 2004;18(2):95-6.

2 Steinkuller PG, Du L, Gilbert C, Foster A, Collins ML, Coats DK. Childhood blindness. J AAPOS. 1999;3(1):26-32.

3 International Committee for the Classification of Retinopathy of Prematurity. The international classification of retinopathy of prematurity revisited. Arch Ophthalmol. 2005; 123(7):991-9.

4 Molinari A, Weaver D, Jalali S. Classifying retinopathy of prematurity. Community Eye Health. 2017;30(99):55-6.

5 Patel J, Patel M, Tucker L, Kalikkot R, Desai J. Do most premature babies get discharged by the expected date of delivery? J Perinatol. 2020;40(5):798-805.

6 Smith LE, Shen W, Perruzzi C, Soker S, Kinose $\mathrm{F}, \mathrm{Xu} \mathrm{X}$, et al. Regulation of vascular endothelial growth factor-dependent retinal neovascularization by insulin-like growth factor-1 receptor. Nat Med. 1999;5(12):1390-5.

7 Hellstrom A, Perruzzi C, Ju M, Engstrom E, Hard AL, Liu JL, et al. Low IGF-I suppresses VEGF-survival signaling in retinal endothelial cells: direct correlation with clinical retinopathy of prematurity. Proc Natl Acad Sci U S A. 2001;98(10):5804-8.

8 Hyland RM, Komlósi K, Alleman BW, Tolnai M, Wood LM, Bell EF, et al. Infantile hemangiomas and retinopathy of prematurity: clues to the regulation of vasculogenesis. Eur J Pediatr. 2013;172(6):803-9.

9 Chang LC, Haggstrom AN, Drolet BA, Baselga E, Chamlin SL, Garzon MC, et al. Growth characteristics of infantile hemangiomas: implications for management. Pediatrics. 2008; 122(2):360-7.

10 Janmohamed SR, Madern GC, de Laat PC, Oranje AP. Educational paper: pathogenesis of infantile haemangioma, an update 2014 (part I). Eur J Pediatr. 2015;174(1):97-103.

11 Olsen GM, Nackers A, Drolet BA. Infantile and congenital hemangiomas. Semin Pediatr Surg. 2020;29(5):150969.

12 Frieden IJ, Haggstrom AN, Drolet BA, Mancini AJ, Friedlander SF, Boon L, et al. Infantile hemangiomas: current knowledge, future directionsProceedings of a research workshop on infantile hemangiomas, April 7-9, 2005, Bethesda, Maryland, USA. Pediatr Dermatol. 2005;22(5):383-406.

13 Amrock SM, Weitzman M. Diverging racial trends in neonatal infantile hemangioma diagnoses, 1979-2006. Pediatr Dermatol. 2013; 30(4):493-4.

14 Drolet BA, Esterly NB, Frieden IJ. Hemangiomas in children. N Engl J Med. 1999;341(3): 173-81.

15 Haggstrom AN, Drolet BA, Baselga E, Chamlin SL, Garzon MC, Horii KA, et al. Prospective study of infantile hemangiomas: demographic, prenatal, and perinatal characteristics. J Pediatr. 2007;150(3):291-4.

16 Munden A, Butschek R, Tom WL, Marshall JS, Poeltler DM, Krohne SE, et al. Prospective study of infantile haemangiomas: incidence, clinical characteristics and association with placental anomalies. Br J Dermatol. 2014; 170(4):907-13.

17 Johnson EF, Davis DM, Tollefson MM, Fritchie K, Gibson LE. Vascular tumors in infants: case report and review of clinical, histopathologic, and immunohistochemical characteristics of infantile hemangioma, pyogenic granuloma, noninvoluting congenital hemangioma, tufted angioma, and Kaposiform Hemangioendothelioma. Am J Dermatopathol. 2018;40(4):231-9.

18 Iacobas I, Phung TL, Adams DM, Trenor CC 3rd, Blei F, Fishman DS, et al. Guidance document for hepatic hemangioma (infantile and congenital) evaluation and monitoring. J Pediatr. 2018;203:294-300.e2.

19 Praveen V, Vidavalur R, Rosenkrantz TS, Hussain N. Infantile hemangiomas and retinopathy of prematurity: possible association. Pediatrics. 2009;123(3):e484-9.

20 Reese AB, Blodi FC. Retrolental fibroplasia; introduction. N Y State J Med. 1952;52(23): 2869-74.

21 Andrews GC, Domonkos AN. Skin hemangioma and retrolental fibroplasia. AMA Arch Derm Syphilol. 1953;68(3):320-2.
22 Greenhouse JM, Szewczyk T. Skin hemangioma and retrolental fibroplasia. AMA Arch Derm. 1956;73(6):568-71.

23 The study examined association of infantile hemangiomas and retinopathy of prematurity in 1997, 2003, and 2006 using the Kids' Inpatient Database (KID), Healthcare Cost and Utilization Project (HCUP), Agency for Healthcare Research and Quality. 2003, 2006, 2009, and 2012.

24 Filippi L, Dal Monte M, Casini G, Daniotti M, Sereni F, Bagnoli P. Infantile hemangiomas, retinopathy of prematurity and cancer: a common pathogenetic role of the $\beta$-adrenergic system. Med Res Rev. 2015;35(3):619-52.

25 Park SY, Kang JH, Jeong KJ, Lee J, Han JW, Choi WS, et al. Norepinephrine induces VEGF expression and angiogenesis by a hypoxia-inducible factor- $1 \alpha$ protein-dependent mechanism. Int J Cancer. 2011;128(10):230616.

26 North PE, Anthony DC, Young TL, Waner M, Brown HH, Brodsky MC. Retinal neovascular markers in retinopathy of prematurity: aetiological implications. Br J Ophthalmol. 2003; 87(3):275-8

27 Léauté-Labrèze C, Dumas de la Roque E, Hubiche T, Boralevi F, Thambo JB, Taïeb A. Propranolol for severe hemangiomas of infancy. N Engl J Med. 2008;358(24):2649-51.

28 Bührer C, Bassler D. Oral propranolol: a new treatment for infants with retinopathy of prematurity? Neonatology. 2015;108(1):49-52.

29 Amir J, Metzker A, Krikler R, Reisner SH. Strawberry hemangioma in preterm infants. Pediatr Dermatol. 1986;3(4):331-2.

30 Kleinman ME, Greives MR, Churgin SS, Blechman KM, Chang EI, Ceradini DJ, et al. Hypoxia-induced mediators of stem/progenitor cell trafficking are increased in children with hemangioma. Arterioscler Thromb Vasc Biol. 2007;27(12):2664-70.

31 Sasaki GH, Pang CY, Wittliff JL. Pathogenesis and treatment of infant skin strawberry hemangiomas: clinical and in vitro studies of hormonal effects. Plast Reconstr Surg. 1984; 73(3):359-70. 